\title{
Convex Sets and Subharmonicity of the Inverse Norm Function
}

\author{
Mohammad Taghi Heydari \\ Department of Mathematics, College of Sciences, Yasouj University, Yasouj 7591874934, Iran \\ Correspondence should be addressed to Mohammad Taghi Heydari; heydari@yu.ac.ir
}

Received 4 November 2021; Revised 10 December 2021; Accepted 30 December 2021; Published 25 January 2022

Academic Editor: Simeon Reich

Copyright $\odot 2022$ Mohammad Taghi Heydari. This is an open access article distributed under the Creative Commons Attribution License, which permits unrestricted use, distribution, and reproduction in any medium, provided the original work is properly cited.

In this paper, we show that in order for a proper compact subset $K$ of plane $\mathbb{R}^{2}$ to be convex, it is necessary and sufficient that inverse norm function be subharmonic.

\section{Introduction}

A function $u: \Omega \longrightarrow[-\infty, \infty)$, where $\Omega$ is a domain in $\mathbb{R}^{n}$, is said to be subharmonic if it is upper semicontinuous, not identically $-\infty$, and satisfies the sub mean value inequality: its average over the boundary of each ball contained in $\Omega$ is greater than or equal to its value at the center.

Let $F$ be a nonempty closed subset of $\mathbb{R}^{n}(n \geq 2)$. By $F^{c}$, we denote the complement of $F$ in $\mathbb{R}^{n}$ and by $\operatorname{co}(F)$ the convex hull of $F$. We define the distance function $d_{F}$ from $F$ by

$$
d_{F}(x)=\operatorname{dist}(x, F)=\inf _{y \in F}\|x-y\|\left(x \in \mathbb{R}^{n}\right),
$$

where $\|$.$\| denotes the Euclidean norm.$

For a given $x$ in $\mathbb{R}^{n}$ and a positive real number $r, S(x, r)$ denotes the sphere centre $x$ and radius $r$ and $B(x, r)$ denotes the open ball of centre $x$ and radius $r$.

Motzkin's Theorem ([1], Theorem 7.8) states that a nonempty closed set $F$ in $\mathbb{R}^{n}$ is convex if and only if every point in $\mathbb{R}^{n}$ has a unique nearest point in $F$. Armitage and Kuran ([3], Theorem 3 ) used this result to prove that $d_{F}(x)$ is subharmonic in $\mathbb{R}^{n}$ if and only if $F$ is convex. Parker [4] prove a local Motzkin-type theorem in order to obtain a local version of Armitage and Kuran's result in the case where $n=$ 2.

Theorem 1. Let $F$ be a nonempty proper closed subset of $\mathbb{R}^{2}$ and let $D$ be a domain such that $F \subset D$. Then, $d_{F}$ is subharmonic in $D$ if and only if $F$ is convex.
He also showed that Theorem 1 does not hold in higher dimensions (see counterexample in [4]).

Now, let $K$ be a compact subset of the plane and $\mu$ be the Lebesgue measure concentrated on $K$, i.e., $\mu=\left.m_{2}\right|_{K}$. Consider the multiplication by $z$ operator $A$, i.e., $(A f)(z)$ $=z f(z)$ for each $f$ in $L^{2}(\mu)$. It is easy to check that $A$ is normal. Let $s \in K$ and put $U_{n}=B(s, 1 / n)$, so $\mu\left(U_{n}\right) \neq 0$. Since $\mu$ is regular, then, $\mu\left(U_{n}\right)<\infty$. Now, define

$$
f_{n}=\frac{1}{\sqrt{\mu\left(U_{n}\right)}} \chi_{U_{n}}
$$

so $\left\|f_{n}\right\|_{2}=1$ and $\left\|(A-s) f_{n}\right\|_{2} \longrightarrow 0$, that is, $s \in \sigma(A)$, where $\sigma(A)$ is the spectrum of $A$. Let $s \in K^{c}$, then, there is an open set $U \subset K^{c}$ with $\mu(U) \neq 0$ and $s \in U$. Define

$$
\psi(z)= \begin{cases}(s-z)^{-1}, & \text { if } z \in U^{c} \\ 0, & \text { if } z \in U\end{cases}
$$

There is $r>0$ such that $B(s, r) \subset U$. If $z \in U^{c}$, then, $(1 /|s-z|)<(1 / r)$. Therefore, $\|\psi\|_{\infty} \leq(1 / r)$ a.e. and so $\psi$ $\in L^{\infty}(\mu)$. Define the operator $T$ on $L^{2}(\mu)$ by $T(f)=\psi f$; then, we have $(s-A) T=T(s-A)=I$ a.e. Thus, $s$ is not in $\sigma(A)$ and so $\sigma(A)=K$. Thus, for $z \in \mathbb{C} / K$, we have (see [5], Proposition 3.9 p.198):

$$
\left\|(z-A)^{-1}\right\|^{-1} \leq d_{K}(z) .
$$


We prove that equality occurs if and only if $K$ is convex. To prove this point, let us recall some definitions. For a bounded linear operator $T$ on a Hilbert space $\mathscr{H}$, the numerical range $W(T)$ is the image of the unit sphere of $\mathscr{H}$ under the quadratic form $x \longrightarrow\langle T x, x>$ associated with the operator. More precisely,

$$
W(T)=\{\langle T x, x\rangle: x \in \mathscr{H},\|x\|=1\} .
$$

Thus, the numerical range of an operator, like the spectrum, is a subset of the complex plane whose geometrical properties should say something about the operator. One of the most fundamental properties of the numerical range is its convexity, stated by the famous Toeplitz-Hausdorff Theorem. The other important property of $W(T)$ is that its closure contains the spectrum of the operator. $W(T)$ is a connected set and for normal operator $N, W \overline{(N)}=c o(\sigma(N))$. Also, for $z \notin W \overline{(T)}, d_{W \overline{(T)}}(z) \leq\left\|(z-T)^{-1}\right\|^{-1}$ (see relation $4.6-7$ of [6]). Therefore, if $A$ is the shift operator defined on $L^{2}(\mu)$, then, we have $W \overline{(A)}=c o(K)$ (Theorem 1.4-4. of [6]). If $K$ is convex, then,

$$
d_{K}(z) \leq\left\|(z-A)^{-1}\right\|^{-1}
$$

It follows, by (4), (6), and Theorem 1 , that

Theorem 2. If $K$ is a compact subset of the plane, then, $K$ is convex if and only if the function $u_{K}: \mathbb{R}^{2} \longrightarrow[0, \infty]$ defined by

$$
u_{K}(z)= \begin{cases}\left\|(z-A)^{-1}\right\|^{-1}, & \text { if } z \in K^{c} \\ 0, & \text { if } z \in K\end{cases}
$$

is subharmonic in $\mathbb{C}$.

\section{Corollary 3.}

$$
\lim \sup _{z \longrightarrow \infty} \frac{1}{\left\|(z-A)^{-1}\right\| \cdot \log |z|}>0 .
$$

Corollary 4. If $z$ is a complex number such that $|z|$ is large enough, then,

$$
0<\frac{\log |z|}{\left\|(z-A)^{-1}\right\|}<1
$$

Corollary 5. (Laplacian). $\Delta u_{K} \geq 0$ in $K^{c}$.

Corollary 6. If $0,1 \notin K$, then,

$$
\left\|(1-A)^{-1}\right\| \cdot \exp \left(\left\|A^{-1}\right\|^{-1}\right) \leq 1 \text {. }
$$

Corollary 7. If $1 \notin K$, then,

$$
0<\left\|(1-A)^{-1}\right\|<1
$$

Let $u$ be subharmonic in $\mathbb{C}$. Define functions $A, B:[0$, $\infty) \longrightarrow[-\infty, \infty)$ by

$$
B(r)=B(r, u)=\inf _{\theta} u\left(r e^{i \theta}\right), A(r)=A(r, h)=\sup _{\theta} u\left(r e^{i \theta}\right)
$$

Then, by the maximum principle, $A(r)$ increases as $r$ increases, but the behavior of $A(r)$ is often erratic. For instance, it can be $\infty$ for some values of $r$. Nevertheless, if $A(r)$ increases not too rapidly, then, there are senses in which the growth of $A$ is controlled by that of $B$.

The order $\lambda$ of a subharmonic function $u$ on $\mathbb{C}$ is defined by

$$
\lambda=\limsup _{r \rightarrow \infty} \frac{\log A(r)}{\log r} .
$$

Littlewood (1908) proved the existence of constants $C$ ( $\lambda)>-\infty$ such that if $u$ is subharmonic in $\mathbb{C}$ with finite order $\lambda$, then,

$$
\limsup _{r \rightarrow \infty} \frac{B(r)}{A(r)} \geq C(\lambda)
$$

Littlewood stated his result for functions of the form $u=\log |f|$ with $f$ entire. His technique still works, though, for general subharmonic $u$. Let us return now to Littlewood's inequality (14), and let $C(\lambda)$ denote also the largest possible such constant. Littlewood showed that for $0 \leq \lambda$ $\leq(1 / 2)$, we have $C(\lambda) \geq \cos (2 \pi \lambda)$. He conjectured (was confirmed) that for $0 \leq \lambda<1$, the correct value should be $C(\lambda)=\cos (2 \pi \lambda)$. An extremal function would be $u_{\lambda}$, defined for $\theta \in[-\pi, \pi]$ by $u_{\lambda}\left(r e^{i \theta}\right)=r^{\lambda} \cos (\lambda \theta)$. Note that for $z \in \mathbb{C}-(-\infty, 0), u_{\lambda}(z)=\operatorname{Re} z^{\lambda}$ is harmonic, so that its Riesz mass is supported on the negative real axis. Also, for fixed $r, u_{\lambda}\left(r e^{i \theta}\right)$ is a symmetric decreasing function of $\theta$. Hence,

$$
B\left(r, u_{\lambda}\right)=u_{\lambda}(-r)=\cos (\pi \lambda) u_{\lambda}(r)=\cos (\pi \lambda) A\left(r, u_{\lambda}\right)
$$

so that the ratio $B(r) / A(r)$ is constant when $u=u_{\lambda}$. For more details, see [7].

Remark 8. Let $w=e^{2 \pi i / n}, n \geq 2, T=\operatorname{co}\left\{1, w, \cdots, w^{n-1}\right\}=$ the convex hull of $\left\{1, w, \cdots, w^{n-1}\right\}$ and $K=r_{0}$.T for some $r_{0}>$ 0 . For the function $u=u_{K}$ defined in Theorem 2, we have $A(r)=r-r_{0} \cos (\pi / n)$ and $A(r)=r-r_{0}$, and then,

$$
\lambda=\lim _{r \longrightarrow \infty} \frac{\log \left(r-r_{0} \cos (\pi / n)\right)}{\log r}=1
$$


Also,

$$
\frac{B(r)}{A(r)}=\frac{r-r_{0}}{r-r_{0} \cos (\pi / n)},
$$

and hence, $C(1)=1$.

\section{Data Availability}

No data was generated or analyzed during the current study.

\section{Conflicts of Interest}

The author declares that they have no conflicts of interest.

\section{References}

[1] F. A. Valentine, Convex Sets, McGraw-Hill, New York, 1964.

[2] F. J. Narcowich, "Analytic properties of the boundary of the numerical range," Indiana University Mathematics Journal, vol. 29, no. 1, pp. 67-77, 1980.

[3] D. H. Armitage and Ü. Kuran, "The convexity of a domain and the superharmonicity of the signed distance function," Proceedings of the American Mathematical Society, vol. 93, no. 4, pp. 598-600, 1985.

[4] M. J. Parker, "Convex sets and subharmonicity of the distance function," Proceedings of the American Mathematical Society, vol. 103, no. 2, pp. 503-506, 1988.

[5] J. B. Conway, A course in functional analysis, (II. Edition), Springer, 2019.

[6] K. E. Gustafon and K. M. Rao, The Numerical Range, the Field of Values of Linear Operators and Matrices, Springer, New York, 1997.

[7] W. K. Hayman and P. B. Kennedy, Subharmonic Functions, vol. 1, Academic Press, London, 1976. 